$N 627 / 726$

NASA TN D-1152

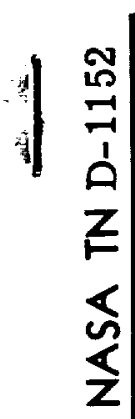

THE TIROS II RADIATION EXPERIMENT

R. A. Hanel and W. G. Stroud

Goddard Space Flight Center

Greenbelt, Md.

NATIONAL AERONAUTICS AND SPACE ADMINISTRATION WASHINGTON October 1961 


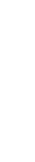




\title{
THE TIROS II RADIATION EXPERIMENT
}

\author{
by \\ R. A. Hanel and W. G. Stroud \\ Goddard Space Flight Center
}

\section{SUMMARY}

The TIROS II meteorological satellite was placed into orbit on November 23, 1960. It contains two television cameras and equipment for a family of electromagnetic radiation experiments, including a medium resolution radiometer. The medium resolution radiometer is a cluster of five sensors which have their optical axes inclined $45^{\circ}$ to the spin axis of the satellite. The spin of TIROS II provides the scanning motion. The five radiometer channels are sensitive to the following spectral bands: 6 to $6.5 ; 8$ to $12 ; 0.2$ to $6 ; 8$ to 30 ; and 0.55 to 0.75 microns. 



\section{CONTENTS}

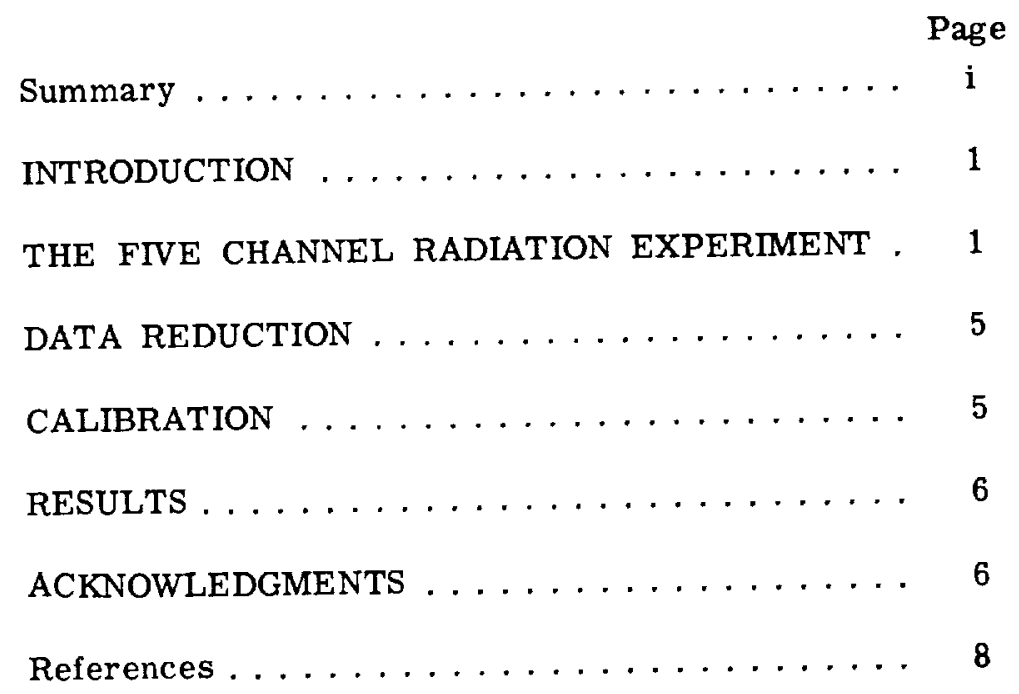





\title{
THE TIROS II RADIATION EXPERIMENT *
}

\author{
by \\ R. A. Hanel and W. G. Stroud \\ Goddord Spoce Flight Center
}

\section{INTRODUCTION}

TIROS $\Pi$, the meteorological satellite $\left(1960 \pi_{1}\right)$ launched on November 23, 1960, carries two television cameras and various detectors to sense infrared emission and reflected solar radiation from the earth. The systems aspect of the radiation experiments, the design of the radiometers, and the physical significance of the measurements have been reviewed elsewhere (References 1, 2,3,4). These other works also contain results of the experiment in the form of analog presentations of individual scan lines; sample data points which show the apparent blackbody temperatures of certain geographical locations; and plotted maps of the radiation emerging from the 8 to 12 micron atmospheric window. Most of these data have been reduced by analog techniques. The main bulk of the information, contained in more than 600 reels of magnetic tape, each 5000 feet long, has to be processed by computers. Hand analyses of samples must precede automatic data reduction, mainly because of the need to judge the validity of the experiment and confirm proper functioning of the instruments. At the present time there is confidence in the measurements and the computer programs are working satisfactorily. The five radiation maps shown in this paper (Figures 1-5) are pilot runs of the automatic data reduction system.

\section{THE FIVE CHANNEL RADIATION EXPERIMENT}

To give a better understanding of the meaning of the maps presented, the instrument which collected the information will be discussed briefly here. The medium resolution

*Presented at the 2nd International Space Science Symposium sponsored by the Committee on Space Research (COSPAR), Florence, Italy, April 1961. 


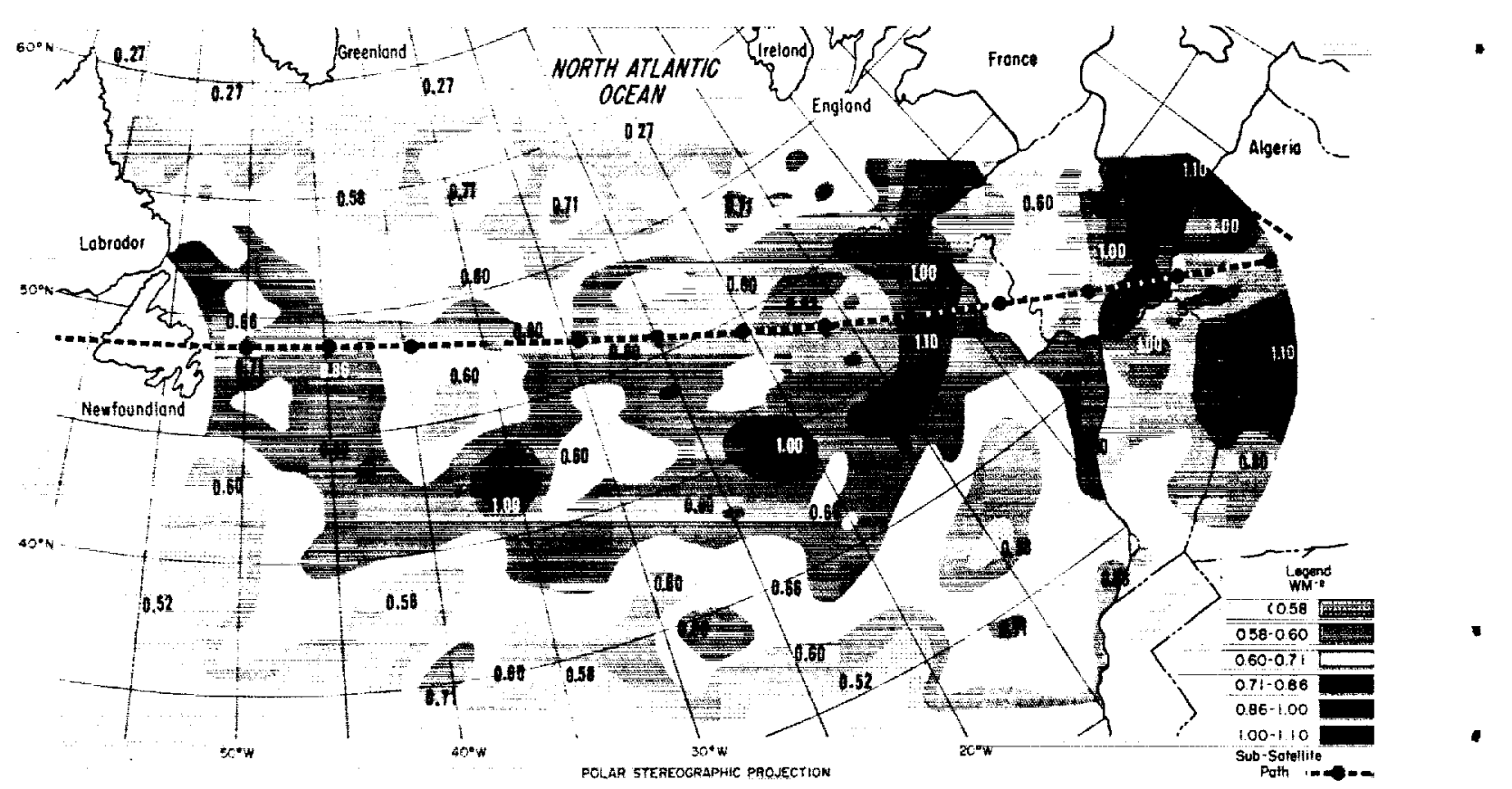

Figure 1 - Radiation mop from Tiros II scanning radiometer: Channel 1 (6.0-6.5 $\mu$ ) Orbit 30, Nov. 25, 1960, 1238 GMT to $1250 \mathrm{GMT}$

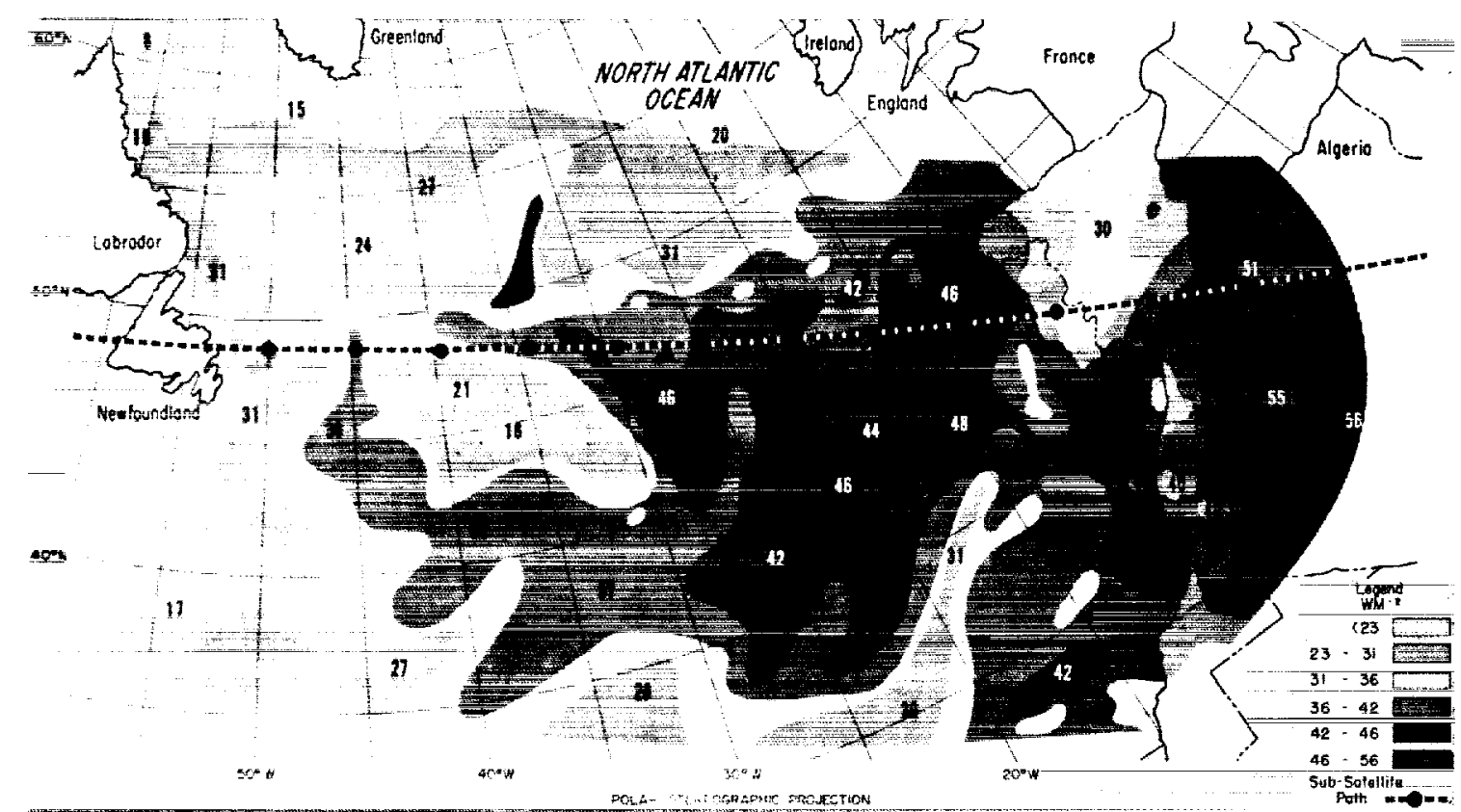

Figure 2 - Radiation map from Tiros II scanning radiometer: Channel 2 (8-12 $\mu$ ) Orbit 30, Nov. 25, 1960, $1238 \mathrm{GMT}$ to $1250 \mathrm{GMT}$ 


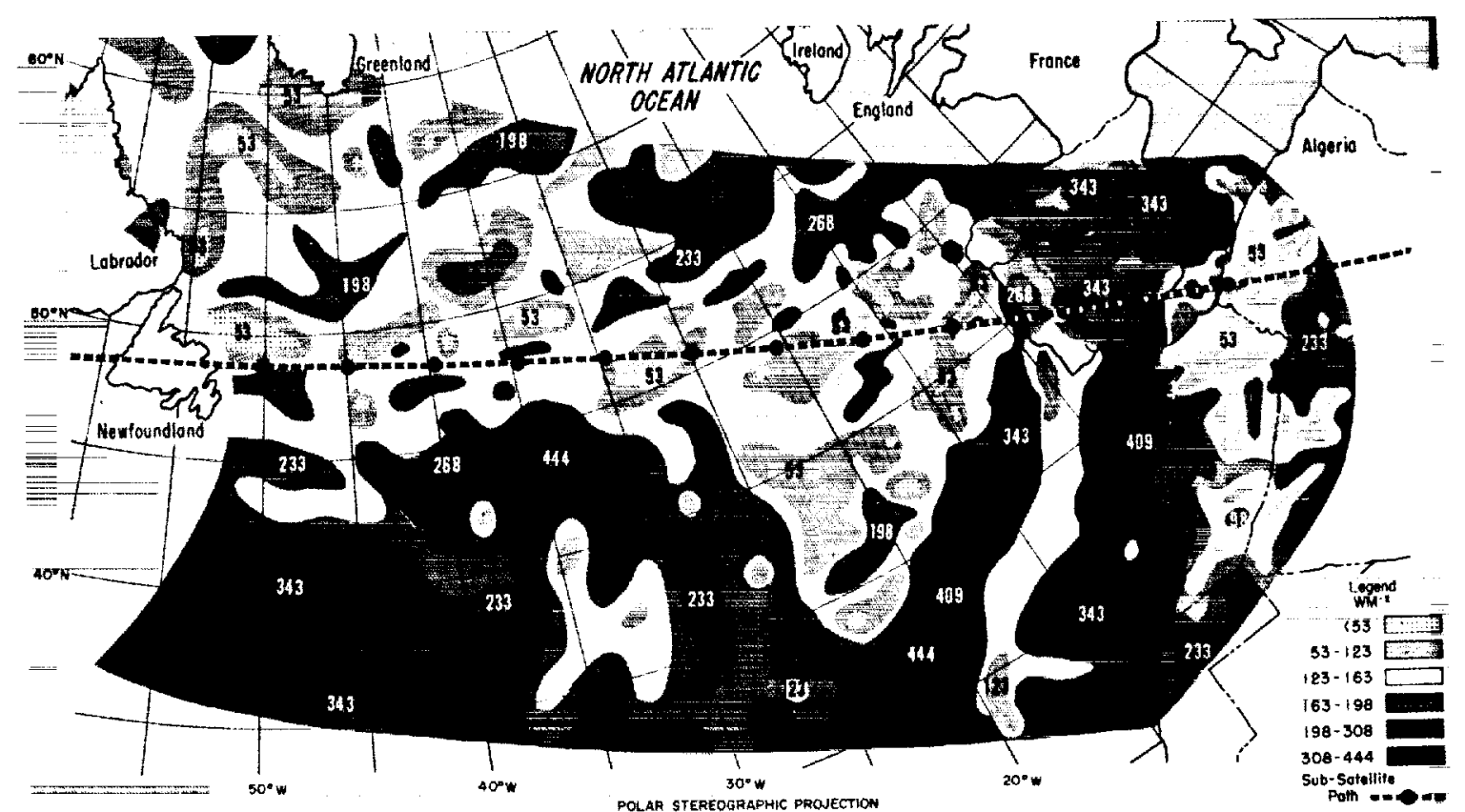

Figure 3 - Radiation map from Tiros II scanning radiometer: Channel 3 (0.2-6.0 $\mu$ ) Orbit 30, Nov. 25, 1960, 1238 GMT to 1250 GMT

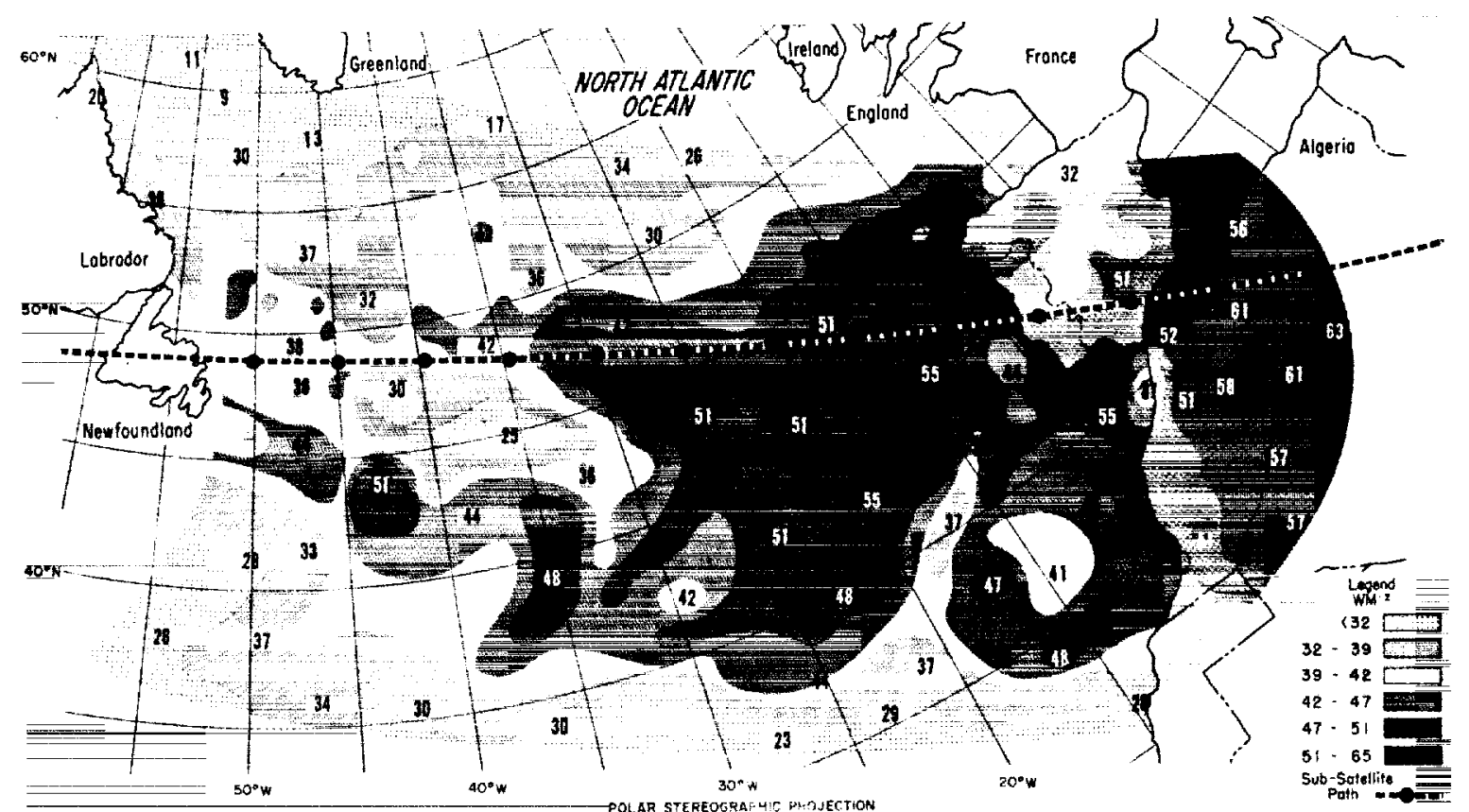

Figure 4 - Radiation map from Tiros II scanning radiometer: Channel 4 (7.8-30 $\mu$ ) Orbit 30, Nov. 25, 1960 , 1238 GMT to $1250 \mathrm{GMT}$ 


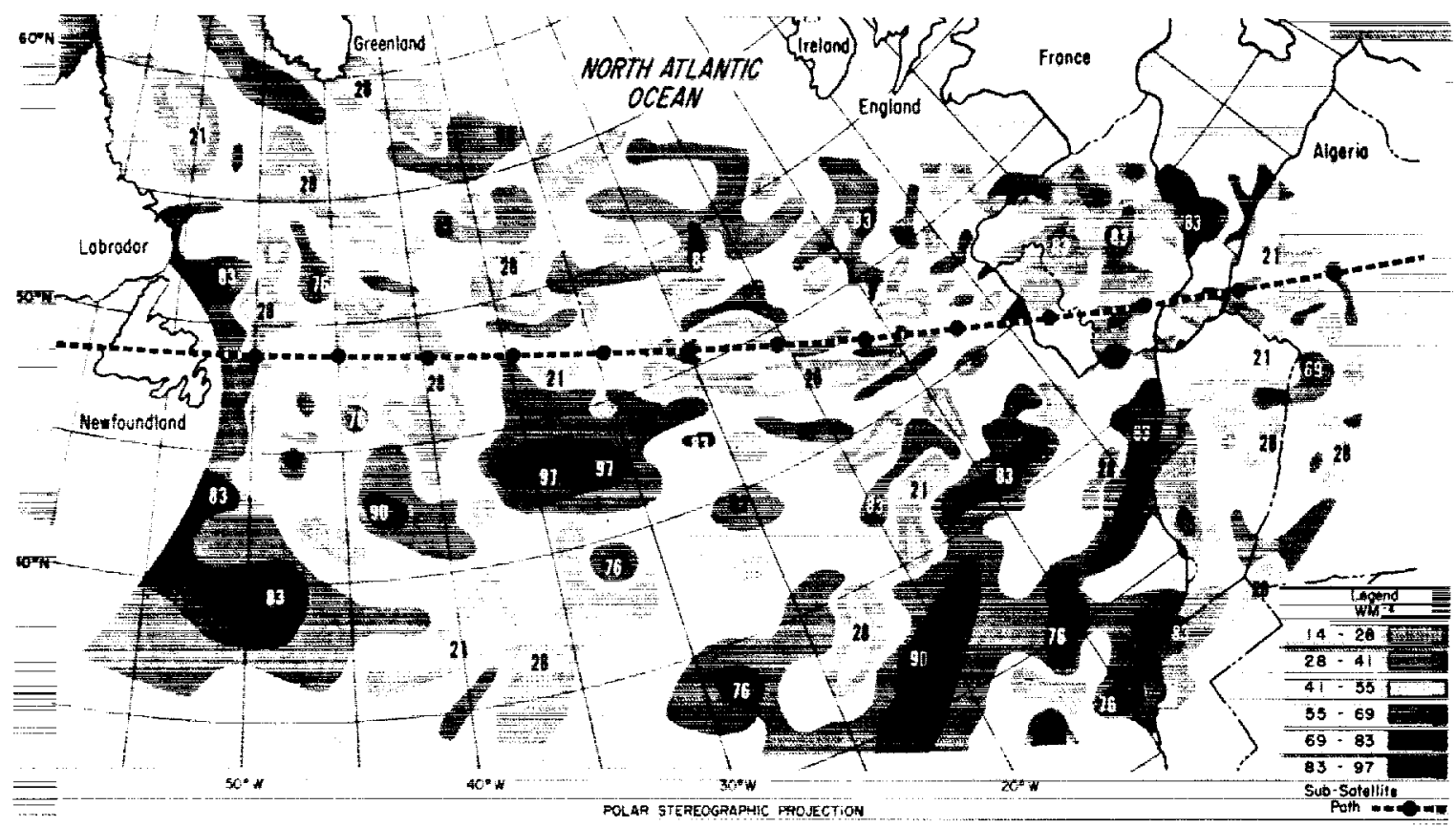

Figure 5 - Radiation map from Tiros II scanning radiameter: Channel $5(0.55-0.75 \mu)$ Orbit 30, Nov. 25, 1960, 1238 GMT to 1250 GMT

radiometer scans earth and outer space as the satellite spins about its axis. Each detector measures the radiation flux within its 5-degree instantaneous field of view and within the spectral range determined by filters and other optical elements. The nominal spectral bandwidths of the five channels are:

(1) 6 to 6.5 microns - water vapor absorption

(2) 8 to 12 microns - atmospheric window

(3) 0.2 to 6 microns - reflected solar radiation

(4) 8 to 30 microns - terrestrial radiation

(5) 0.55 to 0.75 microns - response of television system

The optical axis of the instrument is inclined at 45 degrees to the spin axis of the satellite. The radiometer responds to the flux difference between two diametrically opposite directions. This configuration gives maximum coverage of the earth from a spinstabilized satellite, and uses outer space as a radiation reference. The output of the detectors is stored on magnetic tape in the satellite and transmitted to earth once during each orbit. The receiving stations at Point Mugu, California, and Fort Monmouth, New Jersey, had successfully interrogated TIROS II nearly 2000 times as of this writing. 


\section{DATA REDUCTION}

Each ground station sends the magnetic tape containing the satellite data to the Aeronomy and Meteorology Division, Goddard Space Flight Center, Greenbelt, Maryland. The analog information is then screened and translated into digital form suitable for the IBM 7090 computer of the Meteorological Satellite Laboratory, United States Weather Bureau. The computer output, also on magnetic tape and in digital form, gives radiant emittance in watts per square meter, position of the satellite, geographical location of the area observed, viewing angle, sun angle, and Greenwich mean time for all data points. Figures 1-5 were drawn from computer printouts. Eventually, mapping will be done automatically by contour plotters.

\section{CALIBRATION}

The maps in Figures 1-5 depict radiant emittance $\bar{W}$ in watts per square meter as seen through the instrument's filter:

$$
\bar{W}=\int_{0}^{\infty} w_{\lambda} \phi_{\lambda} d \lambda
$$

where the spectral sensitivity of a channel is called $\phi_{\lambda}$ and $W_{\lambda}$ is the spectral radiant emittance of the area within the field of view. Ideally, $\phi_{\lambda}$ would be constant within the band specified and zero at all other wavelengths. The actual filters used approach this condition. Spectral sensitivities have been discussed in detail in References 2 and 4. During calibration, channels 1, 2, and 4 were exposed to a blackbody of known emittance $W_{\lambda}(T)$. The relations between $\bar{W}$ and the blackbody temperatures are shown in Table 1

Table 1

Radiant Emittances for Various Blackbody Temperatures

\begin{tabular}{|c|c|c|c|}
\hline \multirow{2}{*}{$\begin{array}{c}\text { Blackbody } \\
\text { Temperature } \\
\left({ }^{\circ} \mathrm{K}\right)\end{array}$} & \multicolumn{3}{|c|}{ Radiant Emittance $\overline{\left(\mathrm{w} / \mathrm{m}^{2}\right)}$} \\
\cline { 2 - 4 } & 1 & 2 & 4 \\
\hline 180 & 0.028 & 3.1 & 9.5 \\
200 & 0.10 & 6.5 & 15 \\
220 & 0.28 & 12 & 23 \\
240 & 0.67 & 20 & 35 \\
260 & 1.4 & 32 & 49 \\
280 & 2.6 & 47 & 67 \\
300 & 4.5 & 66 & 89 \\
\hline
\end{tabular}




\section{RESULTS}

The interpretation of the results of channels 3 and 5 also has to take spectral sensitivities into account. A perfectly diffuse surface irradiated by one solar constant (about $1400 \mathrm{w} / \mathrm{m}^{2}$ ) illuminates the detectors of channels 3 and 5 with 1070 and 252 watts per square meter respectively. All scales of the channels show higher accuracy at high radiation levels. Channels 2 and 4 exhibit a better signal-to-noise ratio than does channel 1, and channel 3 data are superior to channel 5 data.

The radiation maps in this paper (Figures 1-5) cover the Atlantic Ocean from Newfoundland to the Iberian Peninsula and North Africa. A surface pressure map and a cloud cover analysis based mainly on ship reports are shown on the same scale in Figures 6 and 7.

The three thermal channels, (Figures 1, 2, and 4) show a general temperature gradient from left to right which is caused by latitudinal and diurnal effects. The local time over Labrador is $9 \mathrm{AM}$ and over France about $1 \mathrm{PM}$. Superimposed over this temperature gradient are synoptic phenomena. The relatively clear area west of Spain can be identified as a warm area by means of channels 2 and 4 . In contrast to this, dense clouds associated with a frontal system (e.g., $35^{\circ} \mathrm{W} 45^{\circ} \mathrm{N}$ ) appear cold in thermal channels 1,2 , and 4 and bright in solar channels 3 and 5 .

As was expected, bright areas, which indicate solid overcase, in channels 3 and 5 are very well correlated to low temperatures in channel 2 which measures radiation from the atmospheric window. This correlation will be used to extend cloud analysis to the nighttime side of the earth, which is unobserved by channels 3 or 5 or by the television cameras.

All maps shown display data which are not corrected for viewing angle and solar zenith angle. Low temperatures at the southern and northern boundary of the scan areas of channels 1 and 4 are caused by limb darkening.

Calculations of the outgoing radiation based on temperature and humidity profiles obtained from balloon soundings plus measurements by a TIROS radiometer on a balloon, will provide additional information on the accuracy of the scales obtained in the TIROS $I$ preflight calibrations.

\section{ACKNOWLEDGMENTS}

The authors would like to thank Mr. L. J. Allison, Goddard Space Flight Center, and Mr. S. D. Soules, U.S. Weather Bureau, for preparing the maps. 


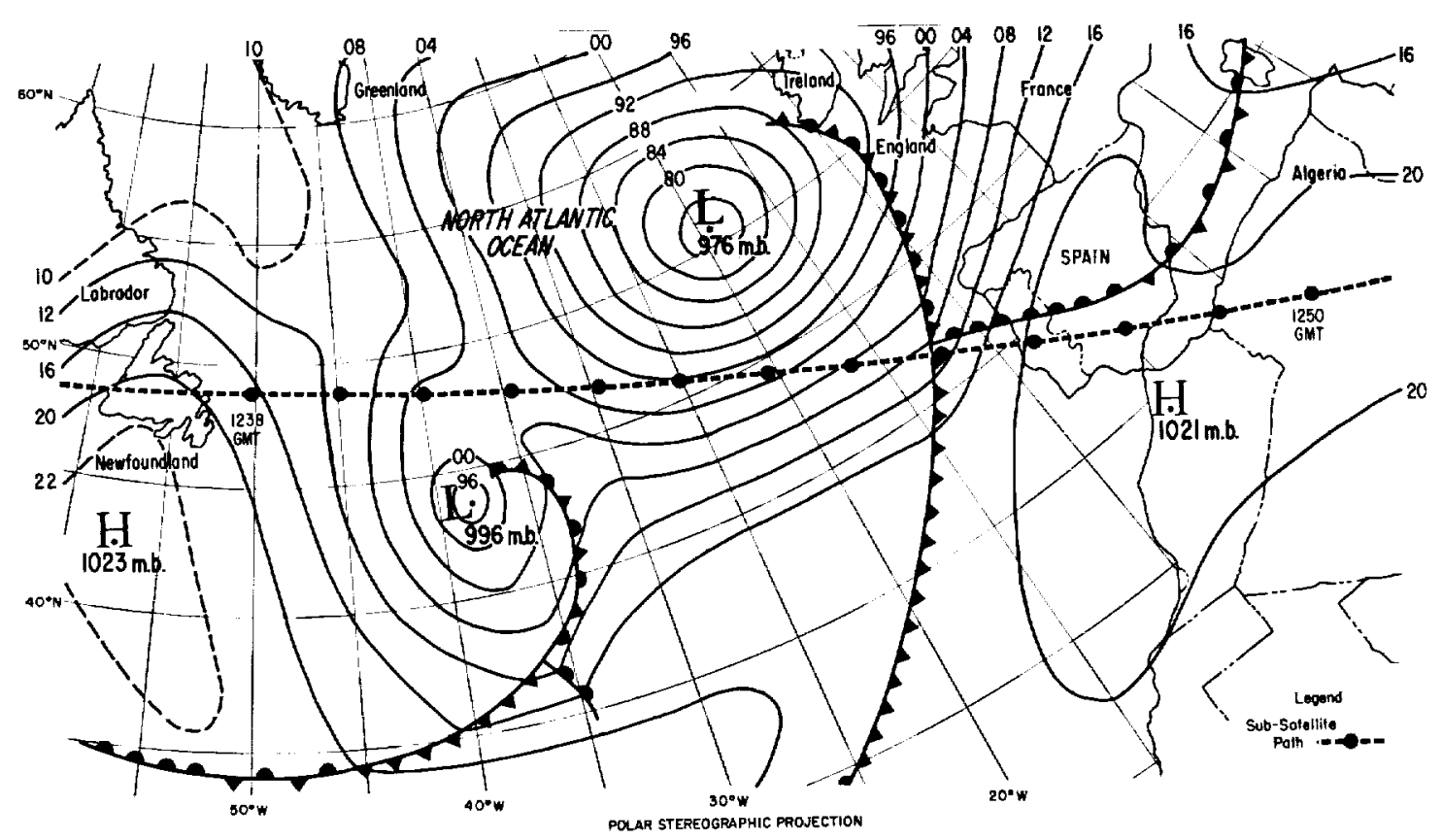

Figure 6. Surface weather chart for 1200 GMT Nov. 25, 1960 showing Orbit 30 of Tiros II

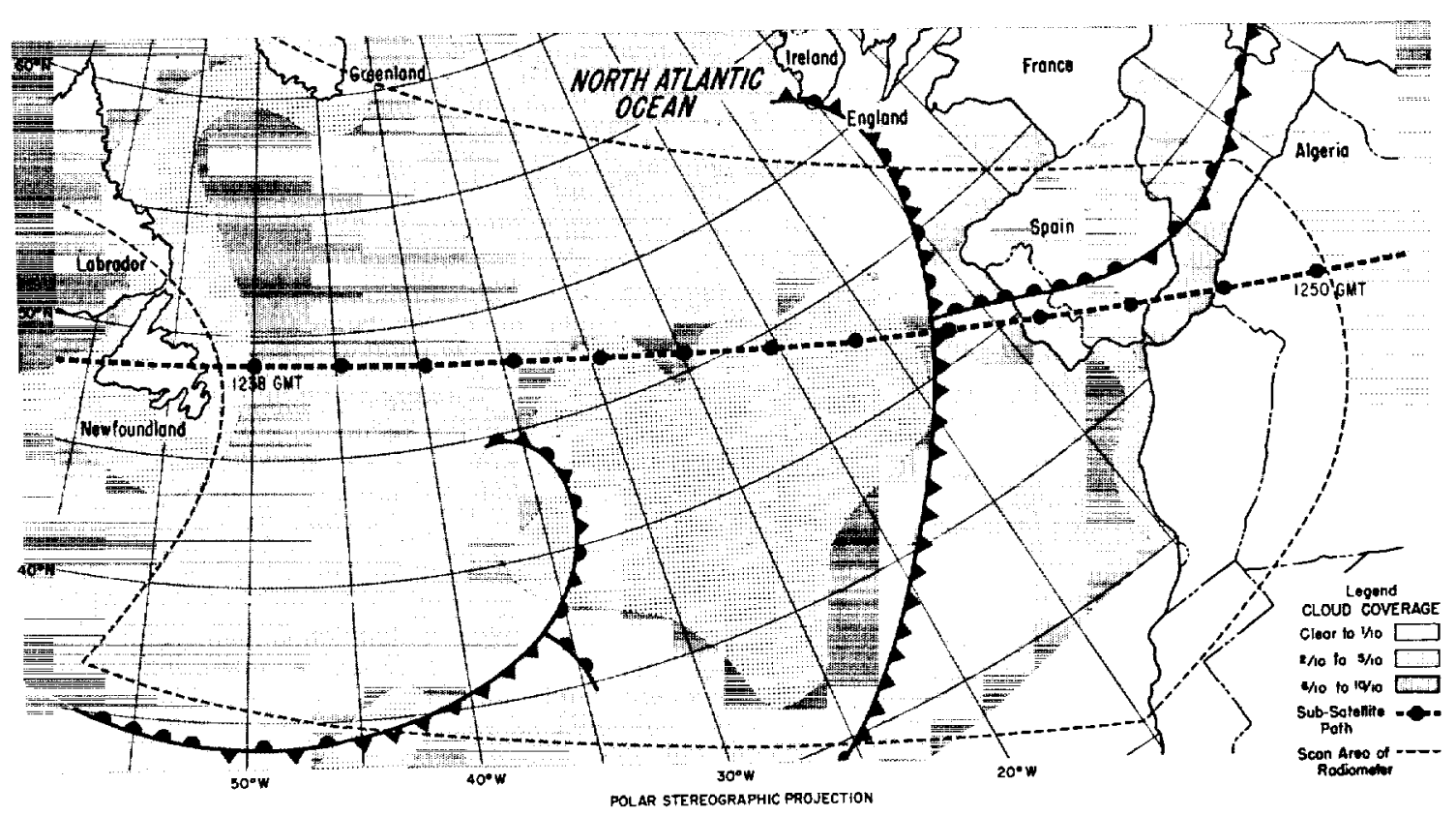

Figure 7 - Nephanalysis and surface weather chart for 1200 GMT Nov. 25, 1960, showing Orbit 30 of Tiros II 
REFERENCES

1. Hanel, R. A., and Stroud, W. G., "Infrared Imaging from Satellites," J. of SMPTE 69(1):25-26, January 1960

2. Bandeen, W. R., Hanel, R. A., et al., "Infrared and Reflected Solar Radiation Measurements from the TIROS II Meteorological Satellite," presented at AMS meeting, New York, January 1961; NASA Technical Note D-1096, 1961

3. Astheimer, R. W., DeWaard, R., and Jackson E. A., paper presented at OSA meeting, Pittsburgh, $\mathrm{Pa}$., March 3, 1961

4. Hanel, R. A., and Wark, D. Q., "Tiros II Radiation Experiment and its Physical Significance," presented at OSA meeting, Pittsburgh, Pa., March 3, 1961; NASA Technical Note D-701, 1961 